\title{
Relation au fournisseur : impératif de multicanalité et gestion des émotions ${ }^{1}$
}

\author{
Béatrice SIADOU-MARTIN²
}

Ghislaine PELLAT ${ }^{3}$

David VIDAL ${ }^{4}$

Fanny POUJOL ${ }^{5}$

\section{Résumé}

Cette recherche s'intéresse aux effets des interactions (en face-à-face et virtuelles) sur les émotions et la satisfaction d'acheteurs en B-to-B. La place des émotions en B-to-B et l'impact des émotions positives sur la satisfaction globale envers le fournisseur sont confirmés. Plus spécifiquement, cette étude montre que si la fréquence des contacts n'a pas d'impact, la nature des contacts a un effet sur les émotions : les interactions en face-à-face génèrent des émotions positives alors que les interactions virtuelles génèrent des émotions négatives.

\begin{abstract}
This research examines the impact of face-to-face and virtual interactions on emotions and satisfaction of the buyer in B-to-B. The role of emotions in the B-to-B context and the influence of positive emotions on the overall satisfaction towards the supplier
\end{abstract}

1 Une version antérieure de cette recherche a fait l'objet d'une présentation lors du colloque de recherche de l'Istec sur le thème "Multicanal et CrossCanal " (12 décembre 2013). Les auteurs tiennent à remercier chaleureusement les évaluateurs anonymes pour leurs encouragements et leurs commentaires très constructifs qui les ont aidés à améliorer l'article.

2 Béatrice SIADOU-MARTIN : Maître de conférences-HDR, Montpellier Research in Management, Université Montpellier II, beatrice.siadou-martin@univ-montp2.fr

3 Ghislaine PELLAT : Maître de conférences, Univ. Grenoble Alpes, CNRS, CERAG, Université de Grenoble, ghislaine.pellat@iut2.upmf-grenoble.fr

4 David VIDAL : Maître de conférences, Montpellier Research in Management, Université Montpellier II, david.vidal@univ-montp2.fr

5 Fanny POUJOL : Maître de conférences-HDR, Montpellier Research in Management, ISEM - Université Montpellier I - Associée à l'INSEEC Paris, fanny.poujol@univ-montp1.fr 
are confirmed. More specifically, the study shows that while the frequency of contacts has no impact, the nature of the contacts has an effect on emotions: face-to-face interactions generate positive emotions while virtual interactions generate negative emotions.

\section{Introduction}

Le baromètre multicanal B-to-B réalisé par Keyrus Management ${ }^{6}$ montre que la vente multicanale en B-to-B progresse en 2013, se rapprochant des scores de la vente en B-to-C. $60 \%$ des entreprises interrogées déclarent vendre en ligne. La part des ventes en ligne au regard des ventes totales représente $16 \%$, ce qui constitue une augmentation de 11 points par rapport à 2011. Ce secteur utilise le e-commerce, les réseaux sociaux et le marketing mobile. 23\% des entreprises en B-to-B relaient leur présence depuis leur site Internet (Thehorel, 2013). De plus en plus de ventes se réalisent par les terminaux mobiles. 21\% des entreprises de l'étude déclarent utiliser le m-mobile, que ce soit via un Smartphone ou une tablette numérique. La vente multicanale en B-to-B est en plein essor.

Si les pratiques multicanales en B-to-B se rapprochent de celles du B-to-C, les questions relatives à l'impact de la vente multicanale se posent, en particulier sur le comportement à tenir vis-à-vis de ces clients professionnels qui eux, vont se soucier du client final. Des chercheurs (Vanheems, 2009 ; Nicholson et Vanheems, 2008) considèrent que la question du multicanal du point de vue de l'acheteur constitue un véritable « challenge » et devient une nécessité pour les entreprises (Neslin et Shankar, 2009). Sous l'effet conjugué de la concurrence et du développement des TIC, les stratégies multicanales multiplient les opportunités et les moyens de contacts avec le consommateur et remettent en cause la vente monocanale classique (Kumar et Venkatesan, 2005 ; Venkatesan et al., 2007).

Lors du processus de vente multicanale, l'acheteur peut choisir entre plusieurs canaux au sein d'un même processus d'achat (Belvaux, 2006). Il utilise alors l'information disponible au moment de l'achat, mais peut aussi inclure une information externe avant la décision d'achat. Son processus d'achat utilise des sources différenciées, ainsi, parle-t-on de stratégie de vente ou d'achat muticanale, chaque canal possédant ses avantages et ses inconvénients (Patricio et al., 2003). Pour les canaux virtuels, ce sont souvent des dimensions utilitaires comme l'information et la facilité d'utilisation qui sont recherchées par les consommateurs. Pour les canaux physiques, ce sont plutôt des dimensions expérientielles et hédoniques qui vont être visées lors de l'achat (Belvaux, 2006). Cependant, le paiement reste encore un élément différenciateur entre les pratiques en B-to-B et en B-to-C. En effet, les conditions tarifaires pratiquées en B-to-B sont encore en faveur de négociations individualisées et en face-à-face ainsi que les services associés personnalisés. C'est parce que la part de la négociation interpersonnelle reste importante, que nous nous sommes intéressés à l'étude des émotions dans les relations interfirmes, en particulier à leur gestion dans le contexte commercial multicanal, en étudiant leur impact sur la satisfaction des clients.

Parmi les travaux qui approchent la question, citons les travaux de Kim et al. (2009) qui mentionnent l'impact du vendeur sur les émotions du client. Komiak et al. (2005) ont 
montré, quant à eux, que le processus de développement de la confiance entre un vendeur et un acheteur est différent selon que l'environnement de vente est réel ou virtuel. Erevelless et Fukawa (2013) vont plus loin en lançant un appel à étudier le rôle joué par l'affect dans ces nouveaux environnements de vente mi-virtuels.

Depuis les travaux fondateurs de Derbaix (Derbaix et Pham, 1989 ; Derbaix et Filser, 2011), l'importance de la composante affective a été soulignée dans la majorité des comportements de consommation. Selon Eagly et Chaiken (2005), l'étude des émotions positives versus émotions négatives est un enjeu pour la recherche du XXI ${ }^{\mathrm{e}}$ siècle. L'étude des affects et des émotions généré(e)s pendant une interaction entre un client et un vendeur, a fait l'objet de plusieurs travaux (Babin et al., 1995 ; Kim et al., 2009 ; Lee et al., 2011). A ce titre, Lee et al. (2011) montrent que les émotions positives ressenties par l'acheteur déterminent la satisfaction vis-à-vis du vendeur.

L'objet de cette recherche est ainsi de mieux comprendre le rôle des émotions sur la satisfaction du client dans un contexte B-to-B, ainsi que l'impact des différents canaux de vente sur les réactions du client. Plus précisément, cette recherche examine les effets de la diversité des canaux utilisés lors des contacts entre un client et son fournisseur. Il s'agit d'étudier l'effet d'un contact multicanal par rapport à un mode de communication comportant un canal unique, sur les émotions du client et sur sa satisfaction vis-à-vis de son fournisseur. Les notions clés de cette recherche sont tout d'abord discutées d'un point de vue théorique. Ces discussions mènent à formuler des propositions de recherche. Ensuite, la méthodologie de l'étude empirique est exposée et justifiée. Enfin, les résultats sont présentés et permettent de souligner de nouvelles voies de recherche.

\section{Multicanalité et émotions ressenties}

\subsection{Quelle est la place accordée aux émotions dans une relation B-to-B ?}

La notion d'affect recouvre un ensemble de processus psychologiques et a été définie comme un ensemble d'états sentimentaux internes à valence positive ou négative, qui incluent les humeurs et les émotions (Westbrook, 1987). Alors que les émotions sont des phénomènes relativement intenses, de courte durée et liés à un objet particulier (stimulus), les humeurs sont des états plus durables qui se manifestent à une plus faible intensité et qui sont indépendants d'un objet spécifique. S'il n'existe pas de définition universellement acceptée, les émotions, qui nous intéressent ici, sont généralement conceptualisées comme des états mentaux résultant de l'évaluation d'un évènement et qui peuvent s'exprimer de façon positive ou négative (Donada et Nogatchewsky, 2009). Les travaux menés en B-to-C montrent que les émotions influencent de nombreux aspects des décisions et des comportements du consommateur. Ainsi, les émotions ont un impact sur la fidélité (Chebat et Slusarczyk, 2005), les comportements qui sont relatifs aux réclamations (Stephens et Gwinner, 1998) mais également sur la satisfaction (Oliver, 1993 ; Graillot, 1998). Les recherches montrent notamment, que les émotions positives ont pour effet d'augmenter la satisfaction des consommateurs alors que les émotions négatives provoquent l'effet inverse (Westbrook, 1987 ; Westbrook et Oliver, 1991). La satisfaction globale est définie comme l'évaluation d'une expérience d'achat, ce jugement 
étant basé sur un niveau de performance du produit ou du service délivré ainsi que sur la qualité d'autres « outputs » (Westbrook et Oliver, 1991).

Bien que les émotions soient aujourd'hui reconnues comme des variables pivots dans l'explication des comportements du consommateur, leur influence dans les échanges entre organisations a été très peu étudiée. Or, les quelques travaux menés à ce sujet mettent en lumière le rôle joué par les états affectifs dans les interactions entre firmes et montrent que les émotions peuvent également façonner les comportements, les processus de décision et les stratégies de négociation des acheteurs professionnels (Andersen et Kumar, 2006).

En comparaison avec des transactions ponctuelles, les relations inter-organisationnelles impliquent généralement une orientation de plus long terme et des interactions interpersonnelles récurrentes entre les individus situés aux frontières des deux firmes (Dwyer et al., 1987). Guidée par une vision économique, la majorité des recherches a analysé les processus cognitifs de ces échanges, se concentrant presque exclusivement sur les aspects rationnels de l'interaction (coûts de transaction, pouvoir, dépendance, engagement...). Considérant qu'un acheteur en B-to-B n'est pas un acteur émotionnel ou impulsif mais un acteur prenant des décisions rationnelles (Voss, 1990) et effectuant des choix souvent issus d'un processus de décision collectif, les travaux existants ont, pour une grande part, ignoré les aspects plus affectifs des relations entre firmes.

Or, cette vision apparaît aujourd'hui contestée, un nombre grandissant de travaux révélant l'importance des éléments affectifs (Wilson, 2000). Si les variables cognitives jouent certes un rôle capital, il est de plus en plus évident que les états affectifs occupent également une place importante au sein du processus d'interaction (Andersen et Kumar, 2006 ; Bagozzi, 2006 ; Kumar, 1997 ; Tähtinen et Blois, 2011 ; Van Dolen et al., 2004). Ainsi, si l'achat en B-to-B doit être conçu comme un amalgame de décisions individuelles, il semble alors possible que ces décisions puissent être influencées par des facteurs affectifs aussi bien que cognitifs (Lynch et De Chernatony, 2004). En effet, les clients en B-to-B, comme les consommateurs, ressentent de nombreux affects lorsqu'ils interagissent avec un vendeur, durant et même après l'interaction (Bagozzi et al., 1999 ; Erevelles et Fukawa, 2013 ; Menon et Dubé, 2000).

Baggozi (2006) suggère que les relations commerciales incluent à la fois des forces rationnelles (processus cognitif) et émotionnelles (processus affectif). La prise en compte de ces deux processus est donc nécessaire pour comprendre la dynamique des partenariats commerciaux. Il va même plus loin en suggérant que les mécanismes de régulation des interactions acheteur-vendeur fonctionnent au travers des émotions sociales interpersonnelles.

Les travaux d'Andersen et Kumar (2006) proposent que les émotions qui émergent au niveau individuel puissent façonner le fonctionnement de la relation lors de son initiation, son développement, sa dissolution et son rétablissement. Partant du constat que les relations inter-organisationnelles reposent sur des interactions interpersonnelles génératrices d'émotions, Täthinen et Blois (2011) montrent que toutes les émotions (à l'exception de l'amour) sont impliquées dans l'évolution d'une relation commerciale, qu'elles soient positives (joie, empathie, fierté, attachement) ou négatives (tristesse, colère, peur, culpabilité, honte). En outre, ces émotions ont des conséquences significa- 
tives sur le comportement des individus impliqués et, par conséquent, sur l'évolution de la relation qui lie leurs organisations.

Sur la base des conclusions issues de recherches menées en marketing B-to-C et des travaux consacrés aux émotions dans le cadre des échanges inter-organisationnels, nous posons la proposition de recherche suivante :

\section{P1 : Les émotions ont un effet sur la satisfaction client.}

\subsection{Le multicanal en B-to-B}

Qu'elle soit purement à distance ou mixte, la vente devient de plus en plus multicanale. Au regard de ces différents canaux d'achat, Vanheems (2007) mentionne que les consommateurs tendent à amplifier leurs volumes d'achat face à ces solutions et à migrer d'un canal à l'autre. L'auteur a identifié ces comportements du consommateur multicanal et recommande des précautions particulières dans les mises en place des politiques commerciales. Elle préconise une gestion individualisée des flux des consommateurs dans chaque canal et entre les canaux. Elle évoque « la mobilité relationnelle » que fournissent ces nouveaux modes (informations comparatives sur les sites Internet, essais en magasin avec conseils, possibilité de ramener le produit s'il y a eu une erreur du côté du client comme de l'organisation) et qui augmente le volume d'achats.

Ces conclusions sont supportées par d'autres travaux (Prasad, 1972 ; Keng, 1984) avant même l'avènement d'Internet sur le quotidien des consommateurs ; ce qui laisse à penser qu'au-delà du canal de distribution choisi selon l'utilité que lui attribue le client, d'autres paramètres interfèrent dans l'achat du client, comme la renommée de la marque, l'habitude d'acheter auprès de la même société et les nombreuses interactions avec cette organisation (Vanheems, 2009). Suivre ce client, croiser les informations le concernant voire provoquer des moments plus rapprochés et personnalisés avec lui, devient source d'innovations de services et d'augmentation de la satisfaction dans une logique du service dominant (Vargo et Lush, 2008), d'autant plus que ce dernier est impliqué dans ses achats et recherche des relations sociales à travers ses achats (Nicholson et Vanheems, 2009). Le comportement d'achat professionnel demande de nombreux conseils techniques, une aide industrielle là aussi personnalisée qui font de la vente multicanale en B-to-B un véritable enjeu. Ceci induit des contacts nombreux et répétés et amène à s'interroger sur la fréquence et la nature des contacts.

Il semble que la fréquence des contacts et leur diversité permettent de mieux répondre aux exigences du client en termes de réactivité (Humphreys et Williams, 1996). Le service associé aux produits est un enjeu compétitif pour les entreprises qui le proposent, selon l'expérience mise en avant par Helfer et Michel (2006). Ces auteurs précisent que la proximité avec le client dans une situation en B-to-B devient performante grâce à une approche multicanale car celle-ci permet la personnalisation du service au client, une interactivité forte avec ce dernier et une fréquence régulière de contacts. Nous formulons ainsi les propositions suivantes :

P2a : La fréquence des contacts a un effet positif sur les émotions positives du client.

P2b : La diversité des contacts a un effet positif sur les émotions positives du client. 


\subsection{Quelles sont les émotions générées par les différents canaux ?}

Les états affectifs associés aux lieux d'achat ont été identifiés dans les travaux de Lichtlé et Plichon (2005) par 6 composantes : le plaisir (degré de bien-être et satisfaction d'une personne), l'activation (sommeil ou surexcitation, données physiologiques) et la dominance (pouvoir de contrôle ou d'influence).

La connaissance de l'impact des canaux virtuels est plus limitée. Selon Szymanski et Hise (2000) puis Evanschitzky et al. (2004), l'e-satisfaction provient de deux facteurs dominants : la navigabilité et le design du site Internet. Bèzes (2014) met en évidence les facteurs communs d'image du site et du magasin et souligne la complémentarité des canaux.

Ces travaux réalisés auprès des consommateurs n'ont pas été étendus dans le domaine de l'achat professionnel. Pour un consommateur, il s'agit d'une situation expérientielle d'achat, alors que des acheteurs du secteur industriel ne vivent pas forcément cette situation de la même manière, étant a priori, plus dégagés personnellement du produit.

Une étude sur la valeur phénoménologique en milieu bancaire (environnement multicanalisé) montre que les clients font davantage appel aux conseillers bancaires dans un contexte où la décision qu'ils doivent prendre est auréolée de risques comme un prêt bancaire, engage le long terme ou lors d'un évènement qui demande des précisions individualisées comme par exemple des réclamations juridiques associées à leurs comptes bancaires (Goudey et Julien, 2011). L'aide apportée par le conseiller constitue alors une source de satisfaction pour les clients. L'intégration des canaux produit une création de valeur très appréciée des clients (Seck, 2013), car il s'agit d'une offre plus complète en termes de services offerts (Van Birgelen et al., 2006), associant plus d'options autour des produits et un accès facilité aux services.

Ces conclusions sont intéressantes pour le contexte étudié dans ce travail dans la mesure où les acheteurs de l'échantillon en B-to-B achètent des produits techniques, scientifiques dont les investissements représentent des montants élevés et qui engagent la rentabilité de l'activité des clients. Ces investissements font souvent appel à des prêts ou des mises à disposition de l'appareil au regard d'une relation commerciale à long terme qui générera de la rentabilité basée sur un approvisionnement régulier de consommables associés à l'appareil.

Seck (2008) dresse une typologie de la nature des contacts, selon qu'ils nécessitent ou non une interaction humaine en distinguant, d'une part, les canaux virtuels et, d'autre part, les canaux physiques. Elle définit les canaux virtuels comme « l'ensemble des moyens de communication utilisant des technologies avancées de l'information et de la communication, des technologies multimédias » et les canaux physiques comme «l'ensemble des moyens de communication avec le client employant des infrastructures physiques (incluant les facilités physiques dans un contexte de service en face-à-face et d'infrastructures logistiques dans un contexte de service logistique) " (Sousa et Voss, 2006). Chaque catégorie de canaux possède ses avantages et ses inconvénients et le choix du canal par le client dépend du type de service (Van Birgelen et al., 2006). Les services routiniers caractérisés par des procédures standards et des décisions relativement simples, sont les plus automatisables 
(Flipo, 1998), alors que les services qui nécessitent une « customization » plus grande et plus complexe privilégient un canal physique.

Compte tenu de la spécificité de notre champ d'étude, le face-à-face paraît plus adapté aux exigences de la clientèle. Nous formulons ainsi les propositions de recherche suivantes :

\section{P3a : Un contact en face-à-face a un effet positif sur les émotions positives du client.}

\section{P3b : Un contact virtuel a un effet négatif sur les émotions positives du client.}

\section{Méthodologie de l'étude empirique}

Pour examiner ces propositions, une enquête par questionnaire a été conduite et les données ont été collectées auprès de 130 acheteurs français travaillant dans le secteur du bio-diagnostic.

\subsection{Choix du terrain, collecte des données et description de l'échantillon}

Le choix du terrain s'est porté sur le secteur du biomédical, et plus particulièrement du bio-diagnostic. La justification de ce choix repose sur les caractéristiques de l'interaction client/fournisseur dans ce secteur. En effet, le processus de vente implique des négociations commerciales parfois longues et aboutit à la proposition d'une offre de service unique pour le client. Il s'agit ainsi d'un service sur-mesure au regard des caractéristiques techniques, financières et de maintenance. Les fournisseurs jouent un rôle important dans la diffusion et la transmission d'informations. Concrètement, ils transmettent aux clients des informations qui concernent l'évolution des pratiques du secteur (technologies et compétences). Ainsi, les contacts entre clients et fournisseurs apparaissent comme nécessaires et relativement fréquents, les commerciaux du fournisseur sont fortement impliqués vis-à-vis de leurs clients.

Après l'obtention d'un accord de principe, un email contenant en pièce jointe le questionnaire est envoyé aux acheteurs. Ce questionnaire comprend trois parties (Annexe 1). Les répondants doivent, dans un premier temps, décrire la relation qu'ils entretiennent avec le fournisseur de leur choix et sur laquelle porte le questionnaire. Ces questions concernent la raison de leur choix, la durée de leur relation, la nature et la fréquence des contacts (mail, téléphone, contact face-à-face et salons professionnels). Puis, les répondants évaluent les émotions ressenties et la prestation de l'entreprise en termes de satisfaction envers le vendeur et l'organisation. Enfin, les caractéristiques sociodémographiques des répondants (genre et position hiérarchique dans l'entreprise) et de l'entreprise (taille et activité) sont mesurées.

L'échantillon est composé d'acheteurs travaillant dans des centres de recherche privés ou publics, ou des laboratoires d'analyses biomédicales. Majoritairement, il s'agit d'hommes (62\%), âgés en moyenne de 43 ans et travaillant dans des petites et moyennes entreprises $(85,3 \%)$. Ils ont une expérience significative puisqu'ils ont une ancienneté supérieure à 6 ans $(61,1 \%)$. La représentativité de l'échantillon est estimée en comparant les caractéristiques des répondants à celles de la population observée. En se basant sur les données 
sur le secteur public et privé, notre échantillon est relativement proche de la population étudiée en termes de genre, âge, ancienneté et taille de l'entreprise.

Nous évoquons dans cette étude, les commerciaux de sociétés qui fournissent à la fois du matériel et des consommables à des professionnels de la santé (biomédical, pas les médecins). Le client final, appelé patient ou encore assuré social, est sensible à l'individualisation de l'information donnée, aux délais de réponse et à la clarté de ces derniers (Tableau 1).

\section{Tableau 1 - Présentation de l'échantillon et de la nature des canaux}

\begin{tabular}{|c|c|c|c|c|c|c|}
\hline & & 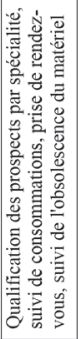 & 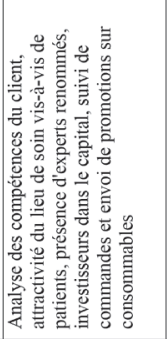 & 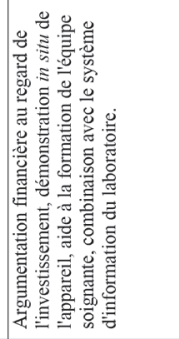 & 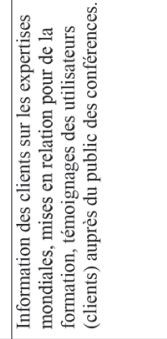 & 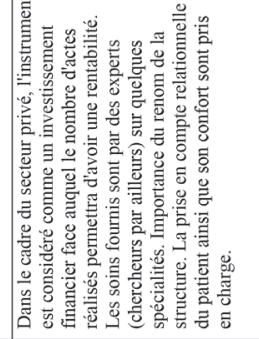 \\
\hline & & 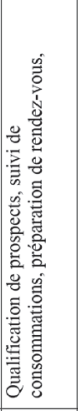 & 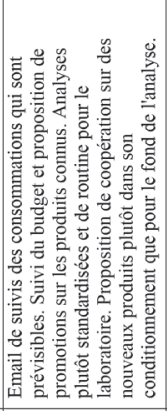 & 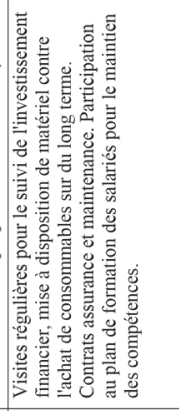 & 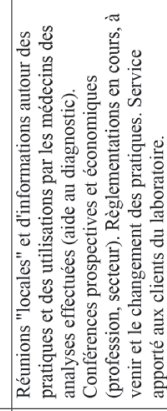 & 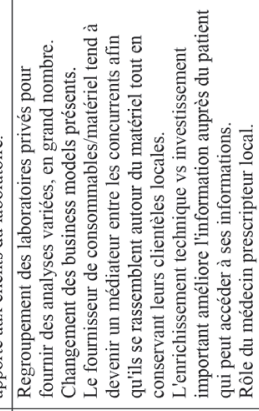 \\
\hline & & 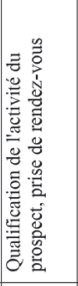 & 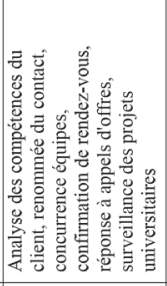 & 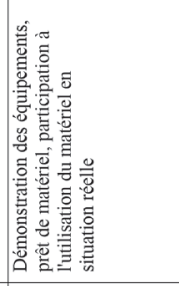 & 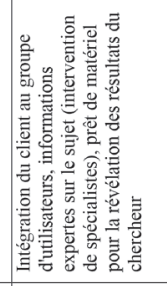 & 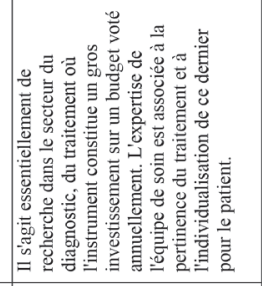 \\
\hline & & 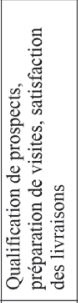 & 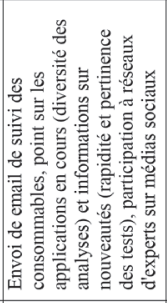 & 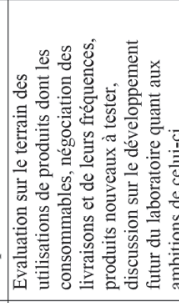 & 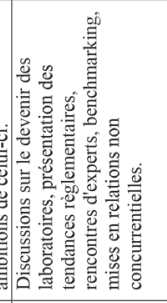 & 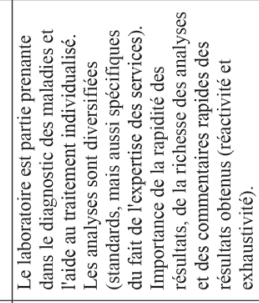 \\
\hline & & & & & نَّ & 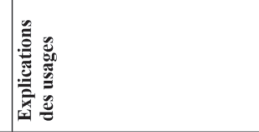 \\
\hline
\end{tabular}




\subsection{Construction du questionnaire et développement des mesures}

Les répondants évaluent, pour chaque canal identifié (face-à-face, Internet, téléphone, salons professionnels), la fréquence des contacts comme suit : jamais ou presque, une à trois fois par an, deux à quatre fois par trimestre, deux à quatre fois par mois, deux à trois fois par semaine, tous les jours ou presque. Au-delà de ces données factuelles, deux scores sont calculés.

Une mesure de la stratégie mono ou multicanale est calculée. Chaque canal identifié est codé 0 (absence d'utilisation du canal) ou 1 (utilisation du canal sans considération de la fréquence). Le score varie ainsi entre 1 et $4: 1$ indique que le fournisseur a une stratégie monocanale et 4 indique que l'entreprise a la stratégie multicanale la plus développée (contact par face-à-face, Internet, téléphone, salons professionnels). Cette mesure est le reflet de l'utilisation des canaux de distribution par le client (et non par l'entreprise).

Une mesure de la fréquence totale des contacts (indépendamment du canal utilisé) est également calculée. Pour un canal donné, les fréquences sont notées de 0 à 6 . La somme des quatre canaux utilisés permet d'arriver à un score compris potentiellement entre 0 et 24 (en pratique, la note minimale est 1 ).

Les émotions sont mesurées avec l'échelle de White et Yu (2005), traduite selon une procédure de rétro-traduction. Quatre items ont permis de mesurer les émotions positives : heureux de cette rencontre (émop1) ; confiant dans l'avenir (émop2) ; positif (émop3) ; agréablement surpris (émop4). Les items des émotions négatives sont : agacé (émon1) ; déçu (émon2) ; coupable (émon3) ; humilié (émon4) ; désagréablement surpris (émon5). La satisfaction est mesurée avec l'échelle établie par Cannon et Perreault (1999).

Les répondants évaluent chaque item avec une échelle de Likert à cinq échelons allant de «pas du tout d'accord » à « tout à fait d'accord ». Afin de tester la validité et la fiabilité de ces échelles, les données sont analysées avec les logiciels SPSS et AMOS. Ces analyses exploratoires et confirmatoires attestent la validité et la fiabilité des échelles. Le coefficient de fiabilité interne (alpha de Cronbach) est de 0,88 pour les émotions positives, de 0,97 pour les émotions négatives et de 0,92 pour la satisfaction globale.

\section{Principaux résultats}

Les résultats sont structurés en trois parties : tout d'abord, une analyse descriptive est conduite (3.1). Ensuite, l'impact des émotions sur la satisfaction est discuté (3.2). Enfin, les liens entre les émotions et la nature ainsi que la fréquence des contacts sont détaillés (3.3).

\subsection{Place des émotions dans les échanges B-to-B}

Préalablement, il nous a paru essentiel d'analyser la place des émotions dans les échanges. Les résultats permettent de confirmer les conclusions des études antérieures (voir Tableau 2). 
Tableau 2 - Moyenne et écart-type des émotions ressenties par un client

\begin{tabular}{|l|c|c|c|c|c|}
\hline \multicolumn{1}{|c|}{ Affect } & $\mathbf{N}$ & Minimum & Maximum & Moyenne & Ecart-type \\
\hline Heureux & 128 & 1 & 5 & 3,81 & 1,033 \\
\hline Confiant & 127 & 1 & 5 & 3,79 & 1,005 \\
\hline Positif & 127 & 1 & 5 & 3,98 & 0,926 \\
\hline Surpris + & 126 & 1 & 5 & 3,06 & 1,033 \\
\hline Agacé & 127 & 1 & 5 & 3,45 & 1,602 \\
\hline Déçu & 126 & 1 & 5 & 3,53 & 1,573 \\
\hline Coupable & 127 & 1 & 5 & 3,60 & 1,783 \\
\hline Humilié & 127 & 1 & 5 & 3,61 & 1,800 \\
\hline Surpris - & 127 & 1 & 5 & 3,65 & 1,744 \\
\hline
\end{tabular}

Toutes les émotions listées semblent être ressenties dans les relations inter-entreprises. Néanmoins, les écarts types plus élevés pour les émotions négatives indiquent que cellesci sont ressenties avec plus de variance ; peut-être, car elles poussent à mettre fin à ces relations.

Ces résultats font écho aux conclusions des travaux analysant le rôle des émotions en B-to-B. Notamment, ils sont convergents avec les résultats des recherches menées par Täthinen et Blois (2011) qui suggèrent que l'ensemble de l'éventail des émotions humaines à l'exception de l'amour, se manifeste dans les relations inter-organisationnelles.

Pour expliquer ceci et mieux comprendre le contexte, prenons le cas d'un client qui appelle plus souvent son fournisseur pour lui parler de son mécontentement à propos de l'usage d'un produit ou d'une livraison mal réalisée, que pour lui dire son contentement ou encore merci. La verbalisation de cette émotion positive tend à se manifester dans une sphère plus restreinte (entourage proche) alors que la verbalisation de l'émotion négative qui se répand, elle, dans une sphère plus large. Le client continuera malgré tout à commander auprès de son fournisseur, l'incitant à corriger l'erreur évoquée.

\subsection{Liens entre les émotions ressenties et la satisfaction}

Comme nous l'avons proposé en P1, les émotions ont une place non négligeable dans un contexte B-to-B, notamment dans leur pouvoir explicatif de la satisfaction globale envers le fournisseur. D’après une régression, nous constatons que les émotions positives impactent fortement la satisfaction (coefficient de régression standardisé $=0.70 ; p=0,00$ ) alors que les émotions négatives n'ont pas d'effet significatif (coefficient de régression standardisé $=0.00 ; \mathrm{p}=0,99$ ).

\subsection{Emotions ressenties en fonction de la nature et de la fréquence des contacts}

Afin de tester la validité de notre proposition liant la fréquence des contacts aux émotions ressenties par le client, une analyse de régression a été menée, en utilisant le scoring de la fréquence totale de contacts (score compris entre 0 et 24). 


\section{Tableau 3 - Impact de la fréquence des contacts sur les émotions}

\begin{tabular}{|c|c|c|c|c|c|}
\hline Emotions positives & Somme des carrés & Degré de liberté & Carré moyen & $\mathrm{F}$ & Sig. \\
\hline Regression & 0,522 & 1 & 0,522 &, 698 & 0,405 \\
\hline Residual & 70,983 & 95 & 0,747 & & \\
\hline Total & 71,505 & 96 & & & \\
\hline Emotions négatives & 4,879 & 1 & 4,879 & 1,801 & 0,183 \\
\hline Regression & 260,111 & 96 & 2,709 & & \\
\hline Residual & 264,990 & 97 & & & \\
\hline Total & \multicolumn{7}{|l|}{} \\
\hline
\end{tabular}

La fréquence globale des contacts n’a pas de lien significatif avec les émotions ressenties par un client, qu'elles soient positives ou négatives (Tableau 3). Ces résultats ne permettent donc pas de valider P2a selon laquelle la fréquence des contacts a un effet positif sur les émotions positives du client.

Pour aller plus loin dans l'analyse, nous avons ensuite examiné l'impact de la diversité des canaux utilisés lors des contacts entre un client et son fournisseur. L'objectif est d'étudier l'effet d'un contact multicanal par rapport à un mode de communication reposant sur un canal unique sur les émotions ressenties par un client. Pour cela, un score a été calculé afin de mesurer le degré de diversité des canaux utilisés par un client : il s'agit du score indiquant la stratégie monocanale (1) ou multicanale $(2,3$ et 4$)$.

\section{Tableau 4 - Impact de la diversité des contacts sur les émotions}

\begin{tabular}{|c|c|c|c|c|c|}
\hline Emotions positives & Somme des carrés & Degré de liberté & Carré moyen & $F$ & Sig. \\
\hline Regression & 0,105 & 1 & 0,105 & 0,139 & 0,710 \\
\hline Residual & 71,400 & 95 & 0,752 & & \\
\hline Total & 71,505 & 96 & & & \\
\hline Emotions négatives & 10,348 & 1 & 10,348 & 3,901 & 0,051 \\
\hline Regression & 254,641 & 96 & 2,653 & & \\
\hline Residual & 264,990 & 97 & & & \\
\hline Total & &
\end{tabular}

La diversité des canaux utilisés par un client n'a pas d'impact significatif sur les émotions positives ressenties par ce client (Tableau 4). En revanche, cette variable a un effet positif sur les émotions négatives (coefficient standardisé $=0,198$ ). La proposition P2b selon laquelle la diversité des contacts a un effet positif sur les émotions positives du client est ainsi invalidée. Les résultats suggèrent que la multiplication des formes de contact entre un client et son fournisseur est génératrice d'émotions négatives. Une stratégie de communication pleinement multicanale aurait donc un effet néfaste en provoquant le déclenchement d'émotions négatives chez le client.

Afin de donner un éclairage sur ce résultat, décrivons la situation où le fournisseur qui subit la pression de sa société pour réaliser ses objectifs de vente pour la période donnée multiplie les appels téléphoniques, emails, visites chez son client afin que celui-ci achète selon les besoins prévisibles. Le client vivra cette situation comme harassante et impro- 
ductive pour leurs échanges ultérieurs. La fréquence des contacts n'a pas l'effet positif escompté car elle est peut-être en décalage avec les attentes du client à ce moment-là. Dans cette situation, c'est peut être au-delà de la fréquence trop élevée, le mauvais choix du moment du contact qui peut être à l'origine d'émotions négatives.

Pour approfondir ce résultat surprenant de prime abord, la nature des contacts a été investiguée. Il ressort de ces analyses que la nature des contacts n'a pas d'effet significatif sur les émotions négatives (Tableaux 5 et 6). Seul le face-à-face est à l'origine d'émotions positives (Tableaux 7 et 8).

\section{Tableau 5 - Impact de la nature des contacts sur les émotions négatives}

\begin{tabular}{|c|c|c|c|c|c|}
\hline & Somme des carrés & Degré de liberté & Carré moyen & F & Sig. \\
\hline Regression & 10,320 & 4 & 2,580 & 0,942 & 0,443 \\
\hline Residual & 254,670 & 93 & 2,738 & & \\
\hline Total & 264,990 & 97 & & & \\
\hline
\end{tabular}

Tableau 6 - Coefficients standardisés selon la nature des contacts sur les émotions négatives

\begin{tabular}{|l|c|c|c|}
\hline & Coefficient standardisé (Beta) & $\mathrm{t}$ & Sig. \\
\hline Téléphone & $-0,094$ & $-0,760$ & 0,449 \\
\hline Internet & 0,199 & 1,701 & 0,092 \\
\hline Face-à-face & 0,082 & 0,730 & 0,467 \\
\hline Conférences & 0,022 & 0,203 & 0,839 \\
\hline
\end{tabular}

La fréquence des contacts sur Internet a un effet positif sur les émotions négatives. Ce résultat étonnant rejoint ceux de l'étude sur la servuction sur Internet (Sabadie et Vernette, 2005). Ces chercheurs font remarquer que la substitution des éléments relationnels de la servuction par une interface homme/machine peut ne pas convenir à tous les types de prestation. Ils recommandent ainsi d'éviter que la technologie ne crée une distance avec le client quand une relation interpersonnelle est attendue.

\section{Tableau 7 - Impact de la nature des contacts sur les émotions positives}

\begin{tabular}{|c|c|c|c|c|c|}
\hline & Somme des carrés & Degré de liberté & Carré moyen & $F$ & Sig. \\
\hline Regression & 5,446 & 4 & 1,361 & 1,896 & 0,118 \\
\hline Residual & 66,059 & 92 & 0,718 & & \\
\hline Total & 71,505 & 96 & & & \\
\hline
\end{tabular}

Tableau 8 - Coefficients standardisés selon la nature des contacts sur les émotions positives

\begin{tabular}{|l|c|c|c|}
\hline & Coefficient standardisé (Beta) & $\mathrm{t}$ & Sig. \\
\hline Téléphone & 0,085 & 0,695 & 0,489 \\
\hline Internet & $-0,019$ & $-0,165$ & 0,870 \\
\hline Face-à-face & 0,222 & 2,019 & 0,046 \\
\hline Conférences & $-0,186$ & $-1,718$ & 0,089 \\
\cline { 2 - 4 }
\end{tabular}


Comme postulé par P3a le face-à-face génère des émotions positives, ce qui confirme les résultats de Schroder et Zaharia (2008) suggérant que les clients utilisent le canal le mieux adapté à leurs besoins. Par ailleurs, les résultats montrent qu'Internet n'a pas d'effet sur les émotions positives du client, invalidant ainsi P3b qui propose qu'un contact virtuel a un effet négatif sur les émotions positives du client.

Dans ce contexte de vente en B-to-B, le canal physique est privilégié. Helfer et Michel (2006) expliquent un lien entre l'orientation relationnelle des individus et le choix du canal pour rentrer en contact avec l'entreprise. Deux groupes d'individus sont identifiés : les relationnels qui ont une prédisposition à développer une relation sociale et les autonomes qui ont tendance à contrôler les tâches qui les lient à l'entreprise. Les individus relationnels privilégient, par exemple, le téléphone tandis que les autonomes citent plutôt le courrier comme moyen de contact. Faut-il en conclure que les acheteurs en B-to-B sont plus relationnels ? Ou alors que la technicité des produits achetés nécessite une réelle adaptation du discours du vendeur à l'univers du client, faisant preuve de créativité ?

Pour mieux comprendre ce résultat, citons le cas où le fournisseur est l'opérateur qui va jouer un rôle de médiateur pour réaliser un projet important dans lequel il fera cohabiter des concurrents. Aujourd'hui, les laboratoires d'analyses médicales se concentrent afin de pouvoir, à terme, bénéficier de l'accréditation par les autorités de remboursement. Un fournisseur a choisi d'anticiper ces évolutions et a permis des investissements importants en instruments (qu'aucun laboratoire ne pouvait faire seul) et d'offrir ainsi, pour tous, la possibilité de réaliser des analyses qu'ils ne faisaient pas jusque-là. Ce modèle original n'a pu se réaliser qu'après de longues réunions, appels téléphoniques et propositions de la part du fournisseur. Le canal de communication privilégié était bien entendu le face-à-face même si toutes les informations étaient disponibles sur le site de chacun des participants et du fournisseur lui-même. La valeur ajoutée de ce projet, gérée et proposée par le fournisseur, repose dans la capacité de dialogue entre les partenaires.

\section{Discussion et voies de recherche}

\subsection{Implications théoriques et managériales}

L'objectif de cette recherche est de tester le rôle des émotions dans la relation entre un fournisseur et son client. Elle introduit ainsi un paradoxe entre la dimension cognitive et rationnelle des échanges en B-to-B et la dimension affective avec l'étude des émotions. Plus précisément, le but de l'étude est d'analyser les émotions ressenties au regard des caractéristiques des échanges entretenus en B-to-B (fréquence et nature des contacts). Elle met en lumière plusieurs points clés.

Emotions et satisfaction en B-to-B. Pendant longtemps, la recherche en marketing B-to-B a adopté une vision instrumentale de l'échange entre firmes, considérant que les relations client-fournisseur sont largement guidées par des décisions rationnelles issues de considérations économiques (pouvoir, dépendance, engagements contractuels, coûts de transactions...). Or, cette vision tend à être remise en question dans la littérature qui suggère que l'évolution des relations inter-organisationnelles nécessite, pour être pleinement comprise, de considérer des éléments plus affectifs. Certains auteurs (ex : 
Bagozzi, 2006) allant même jusqu'à soutenir que les variables affectives opèrent comme de véritables mécanismes de régulation des interactions acheteur/vendeur. Partant de l'hypothèse que les relations inter-firmes ne peuvent être seulement analysées sous un angle économique, cette recherche considère le rôle des éléments affectifs dans l'explication de la performance perçue d'une relation commerciale. Les résultats tendent à confirmer que les émotions ressenties par un client constituent un facteur déterminant dans l'explication de son niveau de satisfaction envers son fournisseur. Notamment, les émotions positives associées à un type de canal (le face-à-face) apparaissent comme des variables déterminantes dans l'explication de la satisfaction du client, celles-ci expliquant $70 \%$ de la satisfaction du client en B-to-B. Ce résultat remet ainsi en question la rationalité des transactions en B-to-B. Il s'agit pourtant de la vente d'instruments techniques et des consommables qui requièrent un bon niveau scientifique de la part des acheteurs. Or, comme en B-to-C, l'achat est influencé par une dimension affective.

Parallèlement, cette recherche met également en lumière l'impact non-significatif des émotions négatives sur la satisfaction. Si ce résultat apparaît surprenant de prime abord, il fait toutefois écho aux recherches qui plaident pour une analyse différenciée des facteurs explicatifs de la satisfaction et de l'insatisfaction. Notamment, la théorie des deux facteurs développée par Herzberg (1959) qui considère la satisfaction et l'insatisfaction comme deux variables indépendantes, influencées par des éléments distincts, semble apporter une piste d'explication fertile. Sur la base des arguments développés dans ces travaux, il apparaît intéressant de s'interroger sur le rôle des émotions dans la formation des deux composantes de la satisfaction. Des études complémentaires ayant pour objectif de tester cette conceptualisation (modèle tétraclasse de Llosa, 1997) semblent dès lors nécessaires pour mieux comprendre le rôle des émotions en B-to-B et définir précisément leur impact sur la satisfaction et l'insatisfaction des clients.

Fréquence et diversité des contacts dans une stratégie multicanale en $B$-to-B. Les résultats montrent que la fréquence des contacts en B-to-B ne provoque ni d'émotions positives ni d'émotions négatives. A l'inverse, la multiplicité des canaux utilisés semble façonner les réactions émotionnelles d'un client d'une manière assez surprenante. Ainsi, les résultats de cette recherche démontrent que le recours à une stratégie multicanale est susceptible de générer le développement d'émotions négatives chez l'acheteur. Dit autrement, si la multiplication des contacts entre un client et un fournisseur n'est pas synonyme d'émotions, la multiplication des formes de contacts peut avoir un effet néfaste sur leur relation en déclenchant l'apparition d'émotions négatives chez l'acheteur. Ces résultats soulèvent ainsi clairement la question de la pertinence d'une stratégie multicanale en B-to-B. En effet, si le multicanal n'a pas d'effet sur les émotions positives et que, parallèlement, il peut générer des émotions négatives, il semble alors tout à fait justifié de s'interroger sur le bien-fondé d'une telle stratégie. Cette étude montre qu'une stratégie multicanale assortie d'un « portefeuille de service » fourni au client, qui selon Wallace et al. (2004) détermine la satisfaction et la fidélité du consommateur, n’est pas la panacée en B-to-B.

Une recommandation forte de ce travail plaide en faveur d'une adaptation des modes de contacts aux personnes. Il revient au vendeur de comprendre ce point majeur qu'est le choix du mode de contact préféré du client et à lui, de demander à son client, son mode de fonctionnement pour s'y adapter. 
Nature des contacts et importance du vendeur en face à face. Ce travail souligne également l'importance du face-à-face et de fait, du vendeur, dans le développement de relations en B-to-B. Ce résultat corrobore les apports de la littérature sur la vente en B-to-B (Biong et Selnes, 1996 ; Humphreys et Williams, 1996) ; le vendeur y est considéré comme un acteur stratégique de la qualité de la relation, l'orientation client apparaissant à la source de cette qualité. D'après une étude sur les comportements relationnels des commerciaux (Pellat et al., 2010), le premier moment où les deux individus se rencontrent serait déterminant du futur de la relation commerciale. Cette première phase repose sur des perceptions sensorielles, notamment, la vue donnant lieu à des émotions. A ce titre, il semble que le face-à-face, par sa nature, soit un mode de contact privilégié. De plus, la rencontre en face-à-face vendeur-acheteur permet des interactions immédiates qui sont sans doute déterminantes dans la création d'une relation commerciale plus forte. Ceci est dû à la possibilité pour un vendeur en B-to-B d'adapter instantanément l'offre du fournisseur ainsi que son comportement, à l'acheteur ou à l'équipe acheteuse. Or la réactivité du vendeur est considérée comme un facteur important de satisfaction client (Humphreys et Williams, 1996). Le rôle du discours du vendeur dans la réassurance qu'il apporte à son client en adaptant la « solution produit » à l'environnement de ce dernier a été mis en lumière. Il y aurait donc, au-delà d'une dimension expérientielle et hédonique de la relation client (Belvaux, 2006), un aspect adaptatif fondamental que seule la servuction peut remplir.

Parallèlement, cette recherche met en lumière un autre résultat tout à fait intéressant. En effet, les résultats montrent que le canal virtuel n'est pas générateur d'émotions positives mais qu'il peut, par contre, entraîner le développement d'émotions négatives chez le client. Ces conclusions font écho à celles de Sabadie et Vernette (2005) qui font état de l'effet négatif que peut avoir la substitution de la relation interpersonnelle par une relation homme/machine. Selon eux, il serait néfaste de remplacer le contact direct par un contact virtuel lorsqu'une interaction en face-à-face est attendue par le client, du fait de la distance que la technologie peut créer. En outre, ces résultats sont cohérents avec les postulats issus de la théorie de la richesse des médias (Daft et al., 1987). En substance, cette théorie suggère que les médias peuvent être classés sur un continuum de la richesse de l'information suivant quatre caractéristiques : (1) la capacité de transmettre un feedback immédiat ; (2) la capacité du message à transmettre des indices multiples tels que le ton de la voix qui facilitent l'interprétation du message ; (3) la capacité d'établir un contact personnalisé et (4) la capacité de transmettre un langage riche et varié. Dans cette perspective, la communication orale est considérée comme étant plus riche que la communication écrite et les médias synchrones plus riches que les médias asynchrones. Il semble alors naturel que les canaux virtuels puissent être générateurs d'émotions négatives dans un contexte B-to-B où la relation interpersonnelle est privilégiée pour apporter des solutions personnalisées aux clients.

\subsection{Limites et voies de recherche}

Bien qu'ils apportent quelques éclairages, ces résultats soulèvent de nombreuses interrogations. La première concerne le concept de satisfaction client multicanale. Comme en B-to-C, il nous semble intéressant d'envisager, non plus une addition de satisfactions monocanales, mais plutôt une satisfaction globale des diverses interactions entre le ou 
les gestionnaires de comptes clés et, le ou les acheteurs. Lors de l'achat « cross canal », c'est l'expérience d'achat globale qui est évaluée.

Par ailleurs, il serait intéressant de s'interroger sur le rôle du vendeur dans une stratégie multicanale. Jeanpert et Salerno (2013) estiment que l'adhésion du personnel au multicanal est primordiale au succès des stratégies multicanales. Définie comme l'acceptation du client multicanal et des actions marketing multicanales, cette notion est sans doute importante dans la compréhension de l'interaction que le vendeur a avec son client. Plus cette acceptation est élevée, meilleure est l'harmonie des actions marketing et la rencontre de service.

Une autre piste de recherche porte sur la dynamique des émotions au fil du temps. Selon Thomas et Diener (1990), la mémoire et l'humeur joueraient un effet important dans la prise de décision d'achat du consommateur. En effet, selon l'émotion (ou les émotions) ressenti(es) au cours de ce processus, les réactions affectives évoluent. Et si ces réactions affectives peuvent être mixtes lors du vécu de la situation, avec le temps, la réaction affective devient unipolaire (positive ou négative), en concordance avec l'humeur dont le client se souvient, revenant à des informations de l'époque. L'examen de la nature des affects au cours du processus est une voie de recherche qui permettrait de mieux comprendre la formation des émotions au cours de l'achat. Pour l'approfondir, il serait aussi intéressant d'étudier si ces émotions ressenties sont le fruit d'un canal particulier, du contenu de l'information délivrée par celui-ci, ou de l'adéquation de ce contenu avec les attentes du client.

Une des limites de cette étude concerne le manque d'information sur les sites Internet évoqués. En effet, ceux-ci correspondent-ils aux attentes des clients ? Il serait intéressant d'approfondir la recherche dans ce sens car la qualité du site et son contenu influencent probablement les réactions du client. Selon Hoffman et Novak (1996), les interactions entre le site et le consommateur sont fondamentales car une bonne organisation du site favorise l'immersion de l'internaute. Par exemple, lors d'une visite de découverte d'un site, l'internaute adopte un comportement de nature « expérientielle »: les interactions doivent lui faciliter l'apprentissage et la maîtrise du site. De plus, le potentiel interactif d'un site permet un échange bi-directionnel et un fort degré de contrôle des clients (Costes, 1999). Ce contrôle influencerait positivement les évaluations de la qualité des services. Si ce contrôle se révèle défaillant, il devient une source de frustration pour l'internaute (Helme-Guizon, 2002). Par exemple, l'échec d'une recherche d'information, ou l'obligation de téléphoner à un conseiller pour obtenir un renseignement, peuvent être vécus comme des éléments très négatifs. Ladwein (2001) souligne le fait que les internautes ont des exigences élevées quant à la qualité des informations sur l'offre et la facilité d'accès à ces informations.

Enfin, l'examen d'autres facteurs explicatifs de la satisfaction sont à intégrer comme variables explicatives ou de contrôle pour mieux comprendre les réactions de l'acheteur en B-to-B. 


\section{Bibliographie}

ANDERSEN P.-H. et KUMAR R. (2006), "Emotions, trust and relationship development in business relationships: A conceptual model for buyer-seller dyads", Industrial Marketing Management, Vol. 35, No. 4, p. 522-535.

BABIN B.-J., BOLES J.-S. et DARDEN W.-A. (1995), "Salesperson Stereotypes, Consumer Emotions, and Their Impact on Information Processing", Journal of the Academy of Marketing Science, Vol. 23, $\mathrm{N}^{\circ}$ 2, p. 94-105.

BAGOZZI R.-P. (2006), "The role of social and self-conscious emotions in the regulation of business-to-business relationships in salesperson-customer interactions", Journal of Business \& Industrial Marketing, Vol. 21, ํ․7, p. 453-457.

BAGOZZI R.-P., GOPINATH M. et NYER P.-U. (1999), "The role of emotions in marketing", Journal of the Academy of Marketing Science, Vol.27, No. 2, p. 184-206.

BELVAUX B. (2006), « Du e-commerce au multicanal. Les différents rôles de l'internet dans l'acte d'achat », Revue Française du Marketing, N$^{\circ} 209$, p. 49-68.

BEZES C. (2014), "Definition and psychometric validation of a measurement index common to website and store images", Journal of Business Research, In press.

BIONG H. et SELNES F. (1996), "The strategic role of the salesperson in established buyer-seller relationships", Journal of Business to Business Marketing, Vol. 3, Nㅜ 3, p. 39-78.

CHEBAT J.-C. et SLUSARCZYK W. (2005), "How emotions mediate the effects of perceived justice on loyalty in service recovery situations: An empirical study", Journal of Business Research, Vol. 58, N 5, p. 664-673.

CANNON J.-P. et PERREAULT W.-D. (1999), "Buyer-seller relationships in business markets", Journal of Marketing Research, Vol. 36, N 4, p. 439-460.

COSTES Y. (1999), "Incentives and Disincentives of Using Internet as an In-home Shopping Tool: An Empirical Study on French Customers", Cahiers de Recherche DMSP.

DAFT R.-L., LENGEL R.-H. et KLEBE TREVINOI L. (1987), "Message equivocality, media selection and manager performance: implications for information systems", Journal MIS Quarterly, Vol. 11, N 3, p. 355-366.

DERBAIX C. et PHAM M.-T. (1989), « Pour un développement des mesures de l'affectif en marketing : Synthèse des prérequis », Recherche et Application en Marketing, Vol. 4, $\mathrm{N}^{\circ} 4$, p. 71-87.

DONADA C. et NOGATCHEWSKY G. (2009), "Emotions in outsourcing. An empirical study in the hotel industry", International Journal of Hospitality Management, Vol. 28, No. 3, p. 367-373.

DWYER R.-F., SCHURR P.-H. et OH S. (1987), "Developing buyer-seller relationships", Journal of Marketing, Vol. 51, No. 2, p. 11-27.

EAGLY A.-H. et CHAIKEN S. (2005), Attitude research in the 21st century: the current state of knowledge, in D. Albarracín, B.-T. Johnson et M.-P. Zanna (coord.), The handbook of attitudes, Mahwah, NJ: Erlbaum, p. 743-767. 
EREVELLES S. et FUKAWA N. (2013), "The Role of Affect in Personal Selling and Sales Management", Journal of Personal Selling and Sales Management, Vol. 33, No. 1, p. 7-24.

EVANSCHITZKY H., GOPALKRISHNAN R.-I., HESSE J. et AHLERT D. (2004), "E-satisfaction: a re- examination", Journal of Retailing, Vol. 80, №3, p. 239-247.

FLIPO J.-P. (1998), « Automatisation des services: de la technologie au marketing », Décisions Marketing, $\mathrm{n}^{\circ}$ 14, p.55- 61.

FORD D. (1982), "The development of buyer-seller relationships in industrial markets”, in H. Håkansson (coord.), International marketing and purchasing of industrial goods: An interaction approach, John Willey \& Sons, p. 288-303.

GOUDEY A. et JULIEN A. (2011), « Mieux comprendre la valeur phénoménologique du multicanal bancaire : le segment grand public », Gestion 2000, Novembre Décembre, p. 49-64.

GRAILLOT L. (1998), « Emotions et comportement du consommateur », Recherche et Applications en Marketing, Vol. 13, N․ 1, p. 5-23.

HÅKANSSON H. (1982), International marketing and purchasing of industrial goods: An interaction approach, John Willey \& Sons, New York, 415 pages.

HELFER J.-P. et MICHEL G. (2006), « La stratégie de contacts multicanal, risques et facteurs clés de succès », Décisions Marketing, N 41, Janvier-Mars, p. 33-41.

HELME-GUIZON A. (2002), « Sources et conséquences de l'irritation ressentie au cours de la navigation sur un site marchand : une étude exploratoire », Actes du $18^{\text {ème }}$ Congrès International de l'Association Française de Marketing, Lille.

HERZBERG F. (1959), “The Motivation to Work”, New York: John Wiley and Sons.

HOFFMAN D.-L. et NOVAK T.-P. (1996), "Marketing in hypermedia computer mediated environments: conceptual foundations", Journal of Marketing, Vol. 60, N 1, p. 50-68.

HUMPHREYS M.-A. et WILLIAMS M.-R. (1996), "Exploring the relative effects of salesperson interpersonal process attributes and technical product attributes on customer satisfaction", Journal of Personal Selling and Sales Management, Vol. 16, N 3, p. 47-57.

JEANPERT S. et SALERNO F. (2013), « Marketing multicanal : Comment favoriser l'adhésion du personnel des magasins ? », Décisions Marketing, № 71, p. 13-30.

KENG K.-A. et EHRENBERG A.-S.-C. (1984), "Pattern of Store Choice", Journal of Marketing Research, Vol. 21, p. 399-408.

KIM J.-E., JU H.-W., et JOHNSON K.-P. (2009), “Sales Associate's Appearance: Links to Consumers' Emotions, Store Image, and Purchases", Journal of Retailing \& Consumer Services, Vol. 16, $\mathrm{N}^{\circ}$ 5, p. 407-13.

KOMIAK S., WEIQUAN W., BENBASAT I. (2005), “Trust Building in Virtual Salespersons Versus in Human Salespersons: Similarities and Differences", E-Service Journal, Vol. 3 $\mathrm{N}^{\circ} 3$, p. 49-63.

KUMAR R. (1997), "The Role of Affect in Negotiations An Integrative Overview", The Journal of Applied Behavioral Science, Vol. 33, No. 1, p. 84-100. 
KUMAR V., VENKATESAN R. (2005), "Who are the multichannel shoppers and how do they perform?: Correlates of multichannel shopping behavior", Journal of Interactive Marketing, Vol. $19 \mathrm{~N}^{\circ}$ 2, p. 44-6.

LADWEIN R. (2001), « L'impact de la conceptualisation des sites de e-commerce sur

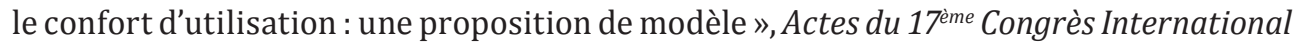
de l'Association Française de Marketing, Caen.

LEE S., COMER L.-B., DUBINSKY A.-J. et SCHAFER K. (2011), "The Role of Emotion in the Relationship Between Customers and Automobile Salespeople",Journal of Managerial Issues, Vol. 23, $\mathrm{N}^{\circ} 2$, p. 206-226.

LICHTLE M.-C., PLICHON V. (2005), « La diversité des états affectifs dans un point de vente », Décisions Marketing, $\mathrm{N}^{\circ} 39$, p. 33-42.

LYNCH J. et DE CHERNATONY L. (2004), "The power of emotion: Brand communication in business-to-business markets", The Journal of Brand Management, Vol. 11, No. 5, p. 403-419.

LLOSA S. (1997), «L'analyse de la contribution des éléments du service à la satisfaction : Un modèle « tétraclasse » », Décisions Marketing, Vol. 10, p. 81-88.

MENON K. et DUBÉ L. (2000), "Ensuring greater satisfaction by engineering salesperson response to customer emotions", Journal of Retailing, Vol. 76, No. 3, p. 285-307.

NESLIN S. et SHANKAR V. (2009), "Key issues in multi-channel customer management: current knowledge and future directions", Journal of Interactive Marketing, Vol. 23, $\mathrm{N}^{\circ} 1$, p. $70-81$.

NICHOLSON P. et VANHEEMS R. (2009), « Orientations d'achat et comportement multicanal du client », Revue Management \& Avenir, $\mathrm{N}^{\circ} 21$, p. 136-156.

OLIVER R.-L. (1993), « Cognitive, affective, and attribute bases of the satisfaction response », Journal of Consumer Research, Vol. 20, $\mathrm{N}^{\circ}$ 3, p. 418-430.

PATRICIO L., FISK R. et CUNHA J. (2003), "Improving satisfaction with bank service offerings: measuring the contribution of each delivery channel", Managing Service Quality, Vol. 13, N 6, p. 471-482.

PELLAT G., POUJOL F. et SIADOU-MARTIN B. (2010), « L'orientation client du vendeur du point de vue du consommateur : Les apports de la théorie de l'attachement », Management \& Avenir, Vo. 31, N 1, p. 246-266.

PRASAD K.-V. (1972), “Correlates of Multistore Food Shopping”, Journal of Retailing, Vol. 48, N 2, p. 74-81.

SABADIE W., VERNETTE E. (2005), « Le management d'une servuction «on line»: quelles caractéristiques et implications pour le marketing ? ", Gestion 2000, Vol. 22, N ${ }^{\circ}$ 6, p. 171-193.

SCHRODER H. et ZAHARIA S. (2008), "Linking multi-channel customer behavior with shopping motives: An empirical investigation of a German retailer", Journal of Retailing \& Consumer Services, Vol. 15, N 6, p. 452-468. 
SECK A.-M. (2008), « La Distribution Multi canal des Services en B to C : Caractéristiques et Challenges », $7^{\mathrm{eme}}$ International Marketing Trends Congress, Venise.

SECK A.-M. (2013), "The issue of Multichannel Integration, A key challenge for Service Firms in a Context of Multichannel Services Distribution", International Business Research, Vol. 6, Nº 2, p. 160-167.

SOUSA R. et VOSS C. (2006), "Service quality in multi-channel service employing virtual channels", Journal of Service Research, Vol. 8, № 4, p. 356-371.

STEPHENS N. et GWINNER K.-P. (1998), "Why don't some people complain? A cognitive-emotive process model of consumer complaint behavior", Journal of the Academy of Marketing Science, Vol. 26, ํㅜ 3, p. 172-189.

SZYMANSKI D.-M. et HISE R.-T. (2000), "E-Satisfaction: An Initial Examination", Journal of Retailing, Vol. 76, $\mathrm{N}^{\circ}$ 3, p. 309-322.

TÄHTINEN J. et BLOIS K.-J. (2011), "The involvement and influence of emotions in problematic business relationships", Industrial Marketing Management, Vol. 40, No. 6, p. 907-918.

THEHOREL L. (2013), « Les entreprises B to B vendent de plus en plus sur le Web », Action Commerciale, 07/01.

THOMAS D.-L. et DIENER E. (1990), "Memory accuracy in the recall of emotions", Journal of Personality and Social Psychology, $\mathrm{N}^{\circ}$ 59, p. 291-297.

VAN BIRGELEN M., DE JONG A. et DE RUYTER K. (2006), "Multi-channel service retailing: the effects of channel performance satisfaction on behavioral intentions", Journal of Retailing, Vol. 82, $\mathrm{N}^{\circ}$ 4, p. 367-377.

VAN DOLEN W., DE RUYTER K. et LEMMINK J. (2004), "An empirical assessment of the influence of customer emotions and contact employee performance on encounter and relationship satisfaction", Journal of Business Research, Vol. 57, No. 4, p. 437-444.

VANHEEMS R. (2007), «Stratégie Multicanal : Valoriser son capital client grâce à une gestion judicieuse des flux de clientèles », Revue française du marketing, $\mathrm{N}^{\circ} 214$, 4/5, p. 61-74.

VANHEEMS R. (2009), « Distribution multicanal, pourquoi les clients mixtes doivent faire l'objet d'une attention particulière ? », Décisions Marketing, $\mathrm{N}^{\circ}$ 55, p. 41-52.

VARGO S. et LUSH R. (2008), "Service-dominant logic: Continuing the Evolution", Journal of the Academy of Marketing Science, Vol. 36, N 1, p. 1-10.

VENKATESAN R., KUMAR V. et RAVISHANKER N. (2007), "Multichannel Shopping: Causes and Consequences", Journal of Marketing, Vol. 71, p. 114-132.

VOSS B. (1990), Cutting through the clutter, Sales and Marketing Management, February, p. 19-20.

WALLACE D., GIESE J.-L. et JOHNSON J.-L. (2004), "Customer retailer loyalty in the context of multiple channel strategies”, Journal of Retailing, Vol. 8, $\mathrm{N}^{\circ}$ 4, p. 249-263.

WESTBROOK R.-A. (1987), "Product/consumption-based affective responses and postpurchase processes", Journal of Marketing Research, Vol. 24, $\mathrm{N}^{\circ}$ 3, p. 258-270. 
WESTBROOK R.-A. et OLIVER R.-L. (1991), "The dimensionality of consumption emotion patterns and consumer satisfaction", Journal of Consumer Research, Vol. 18, $\mathrm{N}^{\circ} 1$, p. 84-91.

WHITE C. et YU Yi-Ting, (2005), "Satisfaction emotions and consumer behavioral intentions", Journal of Services Marketing, Vol. 19, $\mathrm{N}^{\circ}$ 6, p. 411-420.

WILSON D.-F. (2000), "Why divide consumer and organizational buyer behavior?", European Journal of Marketing, Vol. 34, No. 7, p. 780-796. 


\section{Annexe 1 - Questionnaire de recherche}

Votre entreprise évolue dans le secteur du biomédical et entretient des relations commerciales avec plusieurs fournisseurs.

En gardant à l'esprit la relation que vous entretenez avec un fournisseur, pour répondre aux questions suivantes, merci d'écrire votre réponse dans les zones grisées ou de cocher la case qui correspond à votre réponse.

Indiquez la principale raison pour laquelle vous avez choisi de nous parler de cette relation :

C'est le premier fournisseur de mon entreprise en termes de chiffre d'affaires

C'est le fournisseur qui est le plus proche géographiquement de mon entreprise

C'est le fournisseur avec qui mon entreprise a le plus de contacts (téléphone, email, rencontre)

C'est le fournisseur le plus ancien

C'est le fournisseur qui donne le plus d'informations techniques

Autres, précisez :

\section{Depuis quand êtes-vous en relation commerciale avec ce fournisseur?}

Moins d'un an $\square$ Entre un et trois ans (inclus) $\square$ Entre trois et cinq ans (inclus) Plus de 5 ans

Avec quelle fréquence rencontrez-vous le commercial de ce fournisseur ?

\begin{tabular}{|l|c|c|c|c|c|c|}
\hline & $\begin{array}{c}\text { Jamais ou } \\
\text { presque }\end{array}$ & $\begin{array}{c}1 \text { à 3 fois } \\
\text { par an }\end{array}$ & $\begin{array}{c}2 \text { à 4 } \\
\text { fois par } \\
\text { trimestre }\end{array}$ & $\begin{array}{c}2 \text { à 4 fois } \\
\text { par mois }\end{array}$ & $\begin{array}{c}2 \text { à 3 } \\
\text { fois par } \\
\text { semaine }\end{array}$ & $\begin{array}{c}\text { Tous les } \\
\text { " jours » } \\
\text { ou } \\
\text { presque }\end{array}$ \\
\hline Par téléphone & $\square$ & $\square$ & $\square$ & $\square$ & $\square$ & $\square$ \\
\hline Via Internet & $\square$ & $\square$ & $\square$ & $\square$ & $\square$ & $\square$ \\
\hline En face-en-face & $\square$ & $\square$ & $\square$ & $\square$ & $\square$ & $\square$ \\
\hline $\begin{array}{l}\text { Salons professionnels, } \\
\text { conférences }\end{array}$ & $\square$ & $\square$ & $\square$ & $\square$ & $\square$ & $\square$ \\
\hline
\end{tabular}

\begin{tabular}{|l|r|r|r|r|r|}
\hline $\begin{array}{l}\text { Suite aux interactions avec ce vendeur, diriez-vous plu- } \\
\text { tôt que vous vous sentez }\end{array}$ & \multicolumn{4}{|l|}{ Pas du tout } & \multicolumn{4}{|r|}{ d'accord à fait } \\
\hline Heureux de cette rencontre. & $\square 1$ & $\square 2$ & $\square 3$ & $\square 4$ & $\square 5$ \\
\hline Confiant dans l'avenir. & $\square 1$ & $\square 2$ & $\square 3$ & $\square 4$ & $\square 5$ \\
\hline Positif. & $\square 1$ & $\square 2$ & $\square 3$ & $\square 4$ & $\square 5$ \\
\hline Agréablement surpris. & $\square 1$ & $\square 2$ & $\square 3$ & $\square 4$ & $\square 5$ \\
\hline Agacé. & $\square 1$ & $\square 2$ & $\square 3$ & $\square 4$ & $\square 5$ \\
\hline
\end{tabular}




\begin{tabular}{|l|r|r|r|r|r|}
\hline Déçu. & $\square 1$ & $\square 2$ & $\square 3$ & $\square 4$ & $\square 5$ \\
\hline Coupable. & $\square 1$ & $\square 2$ & $\square 3$ & $\square 4$ & $\square 5$ \\
\hline Humilié. & $\square 1$ & $\square 2$ & $\square 3$ & $\square 4$ & $\square 5$ \\
\hline Désagréablement surpris. & $\square 1$ & $\square 2$ & $\square 3$ & $\square 4$ & $\square 5$ \\
\hline
\end{tabular}

\begin{tabular}{|l|r|r|r|r|r|}
\hline $\begin{array}{l}\text { Indiquez votre degré d'accord avec les propositions } \\
\text { suivantes }\end{array}$ & \multicolumn{3}{l}{$\begin{array}{l}\text { Pas du tout } \\
\text { d'accord }\end{array}$} & \multicolumn{3}{l|}{ dout à fait } \\
\hline Travailler avec cette entreprise est une bonne décision. & $\square 1$ & $\square 2$ & $\square 3$ & $\square 4$ & $\square 5$ \\
\hline Dans l'ensemble, je suis satisfait de ce fournisseur. & $\square 1$ & $\square 2$ & $\square 3$ & $\square 4$ & $\square 5$ \\
\hline $\begin{array}{l}\text { Je suis très content de ce que fait ce fournisseur pour mon } \\
\text { entreprise. }\end{array}$ & $\square 1$ & $\square 2$ & $\square 3$ & $\square 4$ & $\square 5$ \\
\hline Mon entreprise est totalement satisfaite de ce fournisseur. & $\square 1$ & $\square 2$ & $\square 3$ & $\square 4$ & $\square 5$ \\
\hline $\begin{array}{l}\text { Si nous devions le refaire, nous choisirions ce fournisseur } \\
\text { à nouveau. }\end{array}$ & $\square 1$ & $\square 2$ & $\square 3$ & $\square 4$ & $\square 5$ \\
\hline
\end{tabular}

Vous êtes : $\quad \square$ un homme $\quad \square$ une femme

Quel est votre âge?

Quelle est votre fonction?

Quelle est votre ancienneté dans cette fonction?

Quelle est la taille de votre entreprise?

Quelle est l'activité principale de votre entreprise? 